\title{
The value of primary and adjuvant radiotherapy for cutaneous squamous cell carcinomas of the head-and-neck region in the elderly
}

Erik Haehl ${ }^{1,2}$, Alexander Rühle ${ }^{1,2}$, Rabea Klink ${ }^{1,2}$, Tobias Kalckreuth ${ }^{1,2}$, Tanja Sprave ${ }^{1,2}$, Eleni Gkika ${ }^{1,2}$, Constantinos Zamboglou ${ }^{1,2}$, Frank Meiß ${ }^{3}$, Anca-Ligia Grosu' ${ }^{1,2}$ and Nils H. Nicolay ${ }^{1,2^{*}}$

\begin{abstract}
Purpose: To examine treatment patterns, oncological outcomes and toxicity rates in elderly patients receiving radiotherapy for cutaneous squamous cell carcinoma (CSCC) of the head-and-neck region.

Material and methods: In this retrospective single-center analysis, locoregional control (LRC), progression-free survival (PFS) and overall survival (OS) of elderly patients $>65$ years with cSCC of the head-and-neck region undergoing radiotherapy between 2010 and 2019 were calculated. The prognostic value of clinicopathological parameters on radiotherapy outcomes was analyzed using the Cox proportional hazards model. In addition, both acute and chronic toxicities were retrospectively quantified according to CTCAE version 5.0.

Results: A total of 69 elderly patients with CSCC of the head-and-neck region with a median age of 85 years were included in this analysis, of whom 21.7\% (15 patients) presented with nodal disease. The majority of patients exhibited a good performance status, indicated by a median Karnofsky performance status (KPS) and Charlson Comorbidity Index (CCl) of $80 \%$ and 6 points, respectively. Radiotherapy was administered as primary (48\%), adjuvant (32\%) or palliative therapy (20\%). 55 patients (79.7\%) completed treatment and received the scheduled radiotherapy dose. Median EQD2 radiation doses were $58.4 \mathrm{~Gy}, 60 \mathrm{~Gy}$ and $51.3 \mathrm{~Gy}$ in the definitive, adjuvant and palliative situation, respectively. 2-year LRC, PFS and OS ranged at $54.2 \%, 33.5$ and $40.7 \%$, respectively. Survival differed significantly between age groups with a median OS of 20 vs. 12 months $(p<0.05)$ for patients aged $65-80$ or above 80 years. In the multivariate analysis, positive lymph node status remained the only significant prognostic factor deteriorating OS (HR $3.73, \mathrm{Cl} 1.54-9.03, p<0.01)$. Interestingly, neither KPS nor CCI impaired survival in this elderly patient cohort. Only 3 patients (4.3\%) experienced acute CTCAE grade 3 toxicities, and no chronic CTCAE grade $2-5$ toxicities were observed in our cohort.
\end{abstract}

Conclusion: Radiotherapy was feasible and well-tolerated in this distinct population, showing the general feasibility of radiotherapy for cSCC of the head-and-neck region also in the older and oldest olds. The very mild toxicities may allow for moderate dose escalation to improve LRC.

Keywords: Cutaneous squamous cell carcinoma, Head-and-neck tumor, Radiotherapy, Elderly, Geriatric

*Correspondence: nils.nicolay@uniklinik-freiburg.de

${ }^{1}$ Department of Radiation Oncology, University of Freiburg - Medical Center, Robert-Koch-Str. 3, 79106 Freiburg, Germany

Full list of author information is available at the end of the article

\section{Introduction}

Non-melanoma skin cancers (NMSC) are among the five most frequent cancer entities with about 3 million original author(s) and the source, provide a link to the Creative Commons licence, and indicate if changes were made. The images or other third party material in this article are included in the article's Creative Commons licence, unless indicated otherwise in a credit line to the material. If material is not included in the article's Creative Commons licence and your intended use is not permitted by statutory regulation or exceeds the permitted use, you will need to obtain permission directly from the copyright holder. To view a copy of this licence, visit http://creativecommons.org/licenses/by/4.0/. The Creative Commons Public Domain Dedication waiver (http://creativeco mmons.org/publicdomain/zero/1.0/) applies to the data made available in this article, unless otherwise stated in a credit line to the data. 
new cases globally per year $[1,2]$. Reported incidences vary widely according to ethnicity, geographic origin and age. With the lack of systematic coverage of NMSC in most cancer registers, incidence is likely underestimated [3]. Cutaneous squamous cell carcinomas (cSCC) account for around 20\% of NMSCs and most often affect elderly patients. A large cohort study of cSCC patients reported a mean age at diagnosis of 70 years and the highest incidence in patients aged above 80 years [4]. With the ongoing demographic changes, the number of patients presenting with cSCC has been increasing rapidly [5]. Apart from rare genetic disorders such as xeroderma pigmentosum, overexposure to UV-light, immunosuppression and chronic scarring constitute major risk factors for cSCC. Wide local surgical excision as the current treatment standard provides excellent cure rates for the majority of cases, while curettage or cryotherapy are alternative treatments that result in similar patient outcomes for small and well-defined cSCCs [6-8]. Nodal or distant metastases develop rarely yet being the main reason for a disease-specific 5-year-mortality of around $2 \%[9,10]$. Increased metastatic risk is reported for deep infiltration, perineural invasion and chronic scarring [11-15].

However, the vast majority of cSCCs present in the head-and-neck region, in which wide local excisions harbor the risk for permanent mutilation [4, 7]. Additionally, distinct facial tumor subsites such as the oral lip and the ear are associated with significantly higher rates of nodal metastases of up to $10 \%$ and therefore more often require multimodal treatment strategies including radiotherapy [11, 12, 16]. Radiotherapy constitutes a curative treatment option if wide local excision is not possible or declined by the patient, and the addition of adjuvant radiotherapy to surgical treatments improves patient outcomes in case of lymph node involvement $[12,17,18]$. However, the benefit of adjuvant radiotherapy for high-risk tumor features such as perineural invasion remains controversial $[18,19]$.

Although cSCC is a disease of the elderly patient, there are only few studies that investigated the role of age regarding treatment outcomes. The newly published American Society for Radiation Oncology (ASTRO) guideline emphasizes the role of radiotherapy in the treatment of cSCC but gives no particular recommendation for the treatment of the elderly, probably due to the lack of evidence [20]. The present analysis seeks to contribute to closing this gap. In this single-center study, we analyzed demographic data, oncologic outcomes and toxicity rates of elderly patients receiving radiotherapy for cSCC between 2010 and 2019 at a major tertiary cancer center. In addition, risk factors correlating with treatment response were investigated in elderly cSCC patients.

\section{Material and methods \\ Patients and treatment}

This retrospective single-center analysis enrolled all patients older than 65 years treated with radiotherapy for histologically confirmed cSCC of the head-andneck region between 2010 and 2019 at the Department of Radiation Oncology, University of Freiburg Medical Center. The study was approved in advance by the institutional ethical review committee (reference no. 551/18). Demographic and clinical data were retrospectively collected from electronic patient files, and pathological data were extracted from the pathology reports.

Treatment for all patients was based on multidisciplinary tumor board recommendations. For photon radiotherapy, patients were immobilized with individually molded thermoplastic masks. Radiotherapy planning was conducted with Oncentra MasterPlan ${ }^{\circledR}$ (Nucletron BV, Veenendaal, The Netherlands) and Eclipse ${ }^{\mathrm{TM}}$ planning softwares (Varian Medical Systems). Depending on the target volume, conformal 3-dimensional radiotherapy (3DRT), intensity-modulated radiotherapy (IMRT) or linear accelerator-generated electron beam radiotherapy were used for treatment (Fig. 1).

\section{Survival and toxicity assessment}

All patients were scheduled for routine follow-up examination at 3 months after radiotherapy and annually thereafter. Additional dermatological follow-up took place in 6-monthly intervals. In case of clinical evidence for local/ locoregional recurrence or distant metastases, followup imaging examinations were carried out at the discretion of the treating physician. Overall survival (OS) was calculated from the completion of treatment to death from any cause, and progression-free survival (PFS) was assessed as the interval between treatment completion and disease progression at any site or death of any cause. Locoregional control (LRC) was defined as the absence of any progression of the primary tumor or the onset or progression of any cervical lymph node metastases. Missing survival data were acquired from the record sections 
of the federal state authorities of Baden-Württemberg through the Comprehensive Cancer Center Freiburg. Acute and chronic toxicities were classified based on the Common Terminology Criteria for Adverse Events (CTCAE), version 5.0. To assess the burden of comorbidity, the age-adjusted Charlson Comorbidity Index (CCI) was used in a modified version with no points given for cSCC itself.

\section{Statistical analyses}

Actuarial OS, PFS and LRC rates were analyzed using the Kaplan-Meier method with the log-rank test to evaluate statistical significance. Univariate and multivariate analyses were performed using the Cox proportional hazards model. $P$-values below 0.05 were considered statistically significant. All statistical analyses were carried out using IBM SPSS Statistics software version 27 (IBM, Armonk, NY, USA).

\section{Results}

Patient and treatment characteristics

A total of 69 patients aged 65 years and above with histologically confirmed cSCCs of the head-and-neck region were included in this analysis (Table 1 ). The most common tumor localizations were nose, ear and cheek $(\mathrm{n}=15,14$ and 9; Additional file 1: Table S1). Median patient age amounted to 85 years (range 66-99 years). Overall patient performance status was satisfactory in this elderly patient cohort with a median Karnofsky performance status (KPS) of 80\% (range 40-100\%) and 80\% of patients having a KPS status of $70 \%$ or higher. Comorbidity burden was moderate with a median score of 6 (range 2-10) in the modified age-adjusted CCI, considering advanced patient age.

The main reason for referral to radiotherapy was local recurrent disease after primary treatment in 43.5\% $(\mathrm{n}=30)$ of cases, followed by primary radiotherapy at initial diagnosis $(\mathrm{n}=26,37.7 \%)$ and metachronous nodal recurrence of $\operatorname{cSCC}(n=13,18.8 \%) .15$ patients $(21.7 \%)$ had clinical lymph node involvement, and only 4 patients (5.8\%) presented with distant metastases. 22 patients (31.9\%) were treated with postoperative radiotherapy after primary resection, mostly due to positive resection margins or remaining tumor $(n=15,68 \%)$.

Radiotherapy was administered in curative intent in 55 patients $(79.7 \%)$ and as palliative treatment in 14 patients (20.3\%) (Table 2; Additional file 1: Table S2). Photon radiotherapy was the main treatment modality for 46 patients (66.7\%); 19 patients $(27.5 \%)$ were treated with electron beam radiotherapy and 4 patients $(5.8 \%)$ received mixed-beam radiotherapy. Integrated and sequential boosts were used in 7 (10.1\%) and 18 (26.1\%)
Table 1 Patient characteristics of elderly patients with CSCC of the head-and-neck region treated with radiotherapy between 2010 and $2019(n=69)$

\begin{tabular}{|c|c|c|}
\hline & $\mathbf{n}$ & $\%$ \\
\hline \multicolumn{3}{|l|}{ Sex } \\
\hline Male & 39 & 56.5 \\
\hline Female & 30 & 43.5 \\
\hline \multicolumn{3}{|l|}{ Age } \\
\hline mean (range) & 84 & $66-99$ \\
\hline $65-80$ & 20 & 29.0 \\
\hline$>80$ & 49 & 71.0 \\
\hline \multicolumn{3}{|l|}{ Presentation } \\
\hline Initial diagnosis & 26 & 37.7 \\
\hline Local recurrence & 30 & 43.5 \\
\hline Nodal recurrence & 13 & 18.8 \\
\hline \multicolumn{3}{|l|}{ T stage } \\
\hline $\mathrm{T} 1$ & 11 & 15.9 \\
\hline $\mathrm{T} 2$ & 6 & 8.7 \\
\hline T3 & 14 & 20.3 \\
\hline T4 & 5 & 7.2 \\
\hline $\mathrm{n} / \mathrm{a}$ & 33 & 47.83 \\
\hline \multicolumn{3}{|l|}{$\mathrm{N}$ stage } \\
\hline No & 23 & 33.3 \\
\hline $\mathrm{N} 1$ & 4 & 5.8 \\
\hline N2 & 10 & 14.5 \\
\hline N3 & 1 & 1.4 \\
\hline $\mathrm{n} / \mathrm{a}$ & 31 & 44.9 \\
\hline \multicolumn{3}{|l|}{ M stage } \\
\hline MO & 23 & 33.3 \\
\hline M1 & 4 & 5.8 \\
\hline$M x$ & 6 & 8.7 \\
\hline $\mathrm{n} / \mathrm{a}$ & 36 & 52.2 \\
\hline \multicolumn{3}{|l|}{ Grading } \\
\hline G1 & 6 & 8.7 \\
\hline G2 & 35 & 50.7 \\
\hline G3 & 19 & 27.5 \\
\hline $\mathrm{n} / \mathrm{a}$ & 9 & 13.0 \\
\hline \multicolumn{3}{|c|}{ R-status (if adjuvant) } \\
\hline Ro & 5 & 22.7 \\
\hline$R x$ & 3 & 13.6 \\
\hline R1 & 10 & 45.5 \\
\hline R2 & 2 & 9.1 \\
\hline $\mathrm{n} / \mathrm{a}$ & 2 & 9.1 \\
\hline \multicolumn{3}{|l|}{ KPS } \\
\hline Median (range) & $80 \%$ & $(40-100 \%)$ \\
\hline $100-90 \%$ & 17 & 24.6 \\
\hline $80-70 \%$ & 38 & 55.0 \\
\hline $60-50 \%$ & 8 & 11.6 \\
\hline$<50 \%$ & 3 & 4.3 \\
\hline $\mathrm{n} / \mathrm{a}$ & 3 & 4.3 \\
\hline \multicolumn{3}{|l|}{$\mathrm{CCl}$} \\
\hline Median (range) & 6 & $(2-10)$ \\
\hline
\end{tabular}


Table 1 (continued)

\begin{tabular}{lll}
\hline & $\mathbf{n}$ & $\%$ \\
\hline$\leq 4$ & 16 & 23.2 \\
5 & 15 & 21.7 \\
6 & 19 & 27.5 \\
7 & 17 & 24.6 \\
$\geq 8$ & 2 & 2.9 \\
\hline
\end{tabular}

KPS Karnofsky performance status, CCI Charlson Comorbidity Index

Table 2 Treatment details for radiotherapy of elderly cSCC patients $(n=69)$

\begin{tabular}{|c|c|c|}
\hline & $\mathrm{n}$ & $\%$ \\
\hline \multicolumn{3}{|l|}{ Radiotherapy } \\
\hline Primary & 33 & 47.8 \\
\hline Adjuvant & 22 & 31.9 \\
\hline Palliative & 14 & 20.3 \\
\hline Photons & 46 & 66.7 \\
\hline Electrons & 19 & 27.5 \\
\hline Both & 4 & 5.8 \\
\hline Boost & 25 & 36.2 \\
\hline Integrated & 7 & 10.1 \\
\hline Sequential & 18 & 26.1 \\
\hline Radiotherapy completed & 55 & 79.7 \\
\hline Radiotherapy discontinued & 14 & 20.3 \\
\hline \multicolumn{3}{|l|}{ Definitive radiotherapy } \\
\hline Median radiation dose (EQD2) & & $58.4 \mathrm{~Gy}$ \\
\hline Median single dose (EQD2) & & $2 \mathrm{~Gy}$ \\
\hline Radiotherapy completed & & $85 \%$ \\
\hline \multicolumn{3}{|l|}{ Adjuvant radiotherapy } \\
\hline Median radiation dose (EQD2) & & $60 \mathrm{~Gy}$ \\
\hline Median single dose (EQD2) & & $2 \mathrm{~Gy}$ \\
\hline Radiotherapy completed & & $77 \%$ \\
\hline \multicolumn{3}{|l|}{ Palliative radiotherapy } \\
\hline Median radiation dose (EQD2) & & $51.3 \mathrm{~Gy}$ \\
\hline Median single dose (EQD2) & & $2.75 \mathrm{~Gy}$ \\
\hline Radiotherapy completed & & $71 \%$ \\
\hline \multicolumn{3}{|l|}{ Reason for non-completion } \\
\hline Tumor progress & & 2 \\
\hline Toxicity & & 7 \\
\hline Comorbidities & & 3 \\
\hline Patient request & & 2 \\
\hline
\end{tabular}

patients, respectively. Median administered radiation doses (EQD2) were 58.4 Gy, 60 Gy and 51.3 Gy in the definitive, adjuvant and palliative setting, respectively.
Initially scheduled radiation doses for the primary and adjuvant setting were both $60 \mathrm{~Gy}$. Fractionation regimes were heterogeneous: 59.4-70 Gy in conventional fractionation was the most frequently used $(\mathrm{n}=35,50.7 \%)$, hypofractionation with 11-13 fractions of 4 Gy was used in 10 cases (14.5\%) (Additional file 1: Table S3). In 27 patients (39.1\%), therapy comprised elective nodal irradiation. 55 patients $(79.7 \%)$ completed the scheduled radiotherapy. Non-completion of radiotherapy was mostly due to treatment-related toxicities $(n=7$; Additional file 1: Table S4), comorbidity $(\mathrm{n}=3)$ and disease progression $(n=2)$ during radiotherapy. Only one patient received concomitant chemotherapy with mitomycin $\mathrm{C}$ and 5-fluorouracil. Median time to the last visit in our clinic was 8 months. Median follow-up calculated with the reversed Kaplan-Meier method for OS was 44 months.

\section{Treatment outcome}

For the whole patient cohort, 2-year rates for OS, PFS and LRC amounted to $40.7 \%, 33.5 \%$ and $54.2 \%$, respectively (Fig. 2). Median OS and PFS were 16 and 8 months, respectively, while median LRC was not reached. 25 (36.2\%) patients experienced locoregional recurrence after therapy, $18(26.1 \%)$ at the primary tumor site and 15 $(21.7 \%)$ as nodal recurrence $(8(11.6 \%)$ patients experienced both local and nodal recurrence). Survival differed significantly between age groups with a median OS of 20 months in patients aged 65 to 80 years compared to only 12 months in patients above 80 years $(p<0.05$, log-rank test). Median PFS was comparable among all age groups and ranged at 8 months for patients up to 80 years versus 7 months for patients older than 80 years $(p=0.13)$. Similarly, LRC did not differ significantly between age groups $(p=0.33)$. Of the analyzed parameters, lymph node involvement had the strongest influence on survival with a median OS of $6(\mathrm{~N}+)$ and 27 months (N0), respectively $(p<0.01)$ (Fig. 3$)$. The prognostic value of nodal involvement was found strongest for the subgroup of patients older than 80 years with a median OS of 34 versus 8 months $(p<0.01)$. For patients aged 65 to 80 years, the negative influence of nodal involvement was not statistically significant, probably due to the small sample size $(p=0.314)$. Positive resection margins prior to radiotherapy were shown to result in a trend towards decreased OS $(p=0.06)$, while T stage, low patient performance or a higher comorbidity burden did not significantly influence $\mathrm{OS}(p=0.18, p=0.76$ and $p=0.66$, respectively). $\mathrm{T}$ stage $(p=0.422)$ and resection margin 

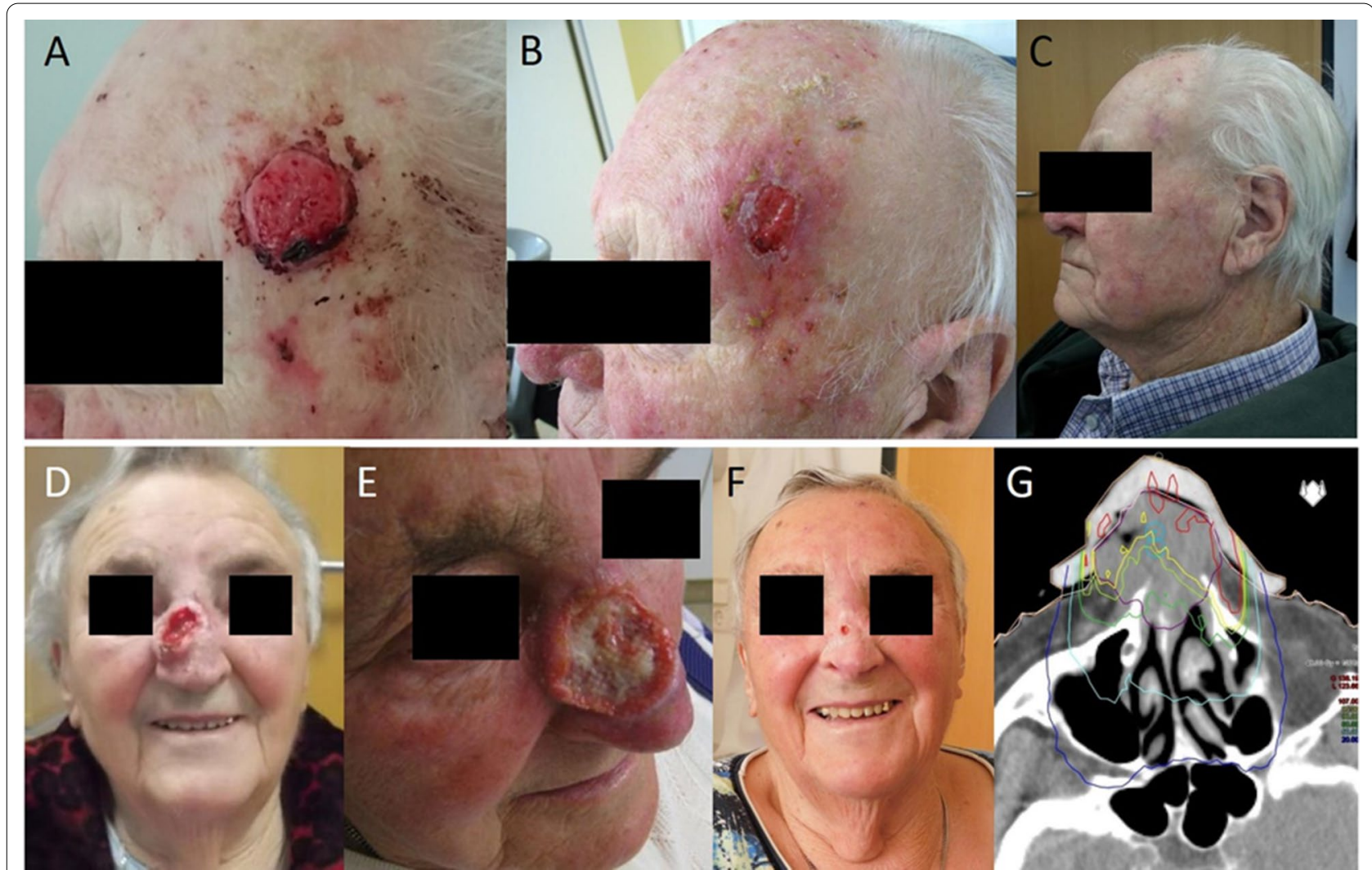

Fig. 1 Representative images of a 94-year old patient with cSCC of the left temple before (A), at the end (B) and 6 weeks after $(\mathbf{C})$ electron beam radiotherapy with 51 Gy-. 88-year old patient with CSCC of the nose before (D), at the end (E) and 5 months after $(\mathbf{F})$ electron beam radiotherapy with $48 \mathrm{~Gy}$. (G) depictures the dose distribution of the electron field

$(p=0.439)$ did not impact LRC, whereas lymphonodal spread was found to significantly deteriorate LRC in the Kaplan-Meier analyses $(p<0.05)$ (Fig. 4).

Interestingly, primary radiotherapy and resection with adjuvant radiotherapy resulted in comparable survival and LRC ( $p=0.43$ and $p=0.88$ ) (Fig. 5), showing the value of adjuvant radiotherapy also for elderly patients in case of incomplete resection. Median LRC was 19 months for patients treated in palliative intend and was not reached for primary curative or adjuvant treatment, although this difference was not statistically significant $(p=0.37)$. OS after palliative radiotherapy amounted to only 11.2 months and was significantly worse than after curative treatment $(p=0.001)$.

Locoregional failure differed for various tumor localizations from no recurrence in seven tumors of the scalp to three out of four tumors of the temporal area (Additional file 2: Figure S1). Due to low case numbers statistical significance was not reached $(p=0.12)$. Incidentally, LRC was markedly better after electron beam radiotherapy with a median of 14 months in the photon cohort and the median not reached in the electron beam cohort $(p=0.004)$. This difference was likely due to a lower prevalence of nodal involvement (5\% versus $28 \%$ ), incomplete resection (16\% versus $33 \%)$ and recurrent disease $(26 \%$ versus $50 \%$ ) in the electron beam cohort (Table 3 ).

In the univariate analysis, an age above 80 years (HR 2.22, CI 1.07-4.60, $p<0.05$ ) and nodal disease (HR 3.68, CI 1.52-8.95, $p<0.01$ ) were found to result in reduced OS, while positive resection margins showed a trend towards impaired OS (HR 5.38, CI 0.70-41.08, $p=0.10$ ). In contrast, both patient performance status (HR 1.10, CI $0.60-2.00, p=0.76$ ) and comorbidity burden (HR 1.14, CI $0.63-2.05, p=0.67$ ) were found to have no prognostic influence in our cohort. In the multivariate analysis, 

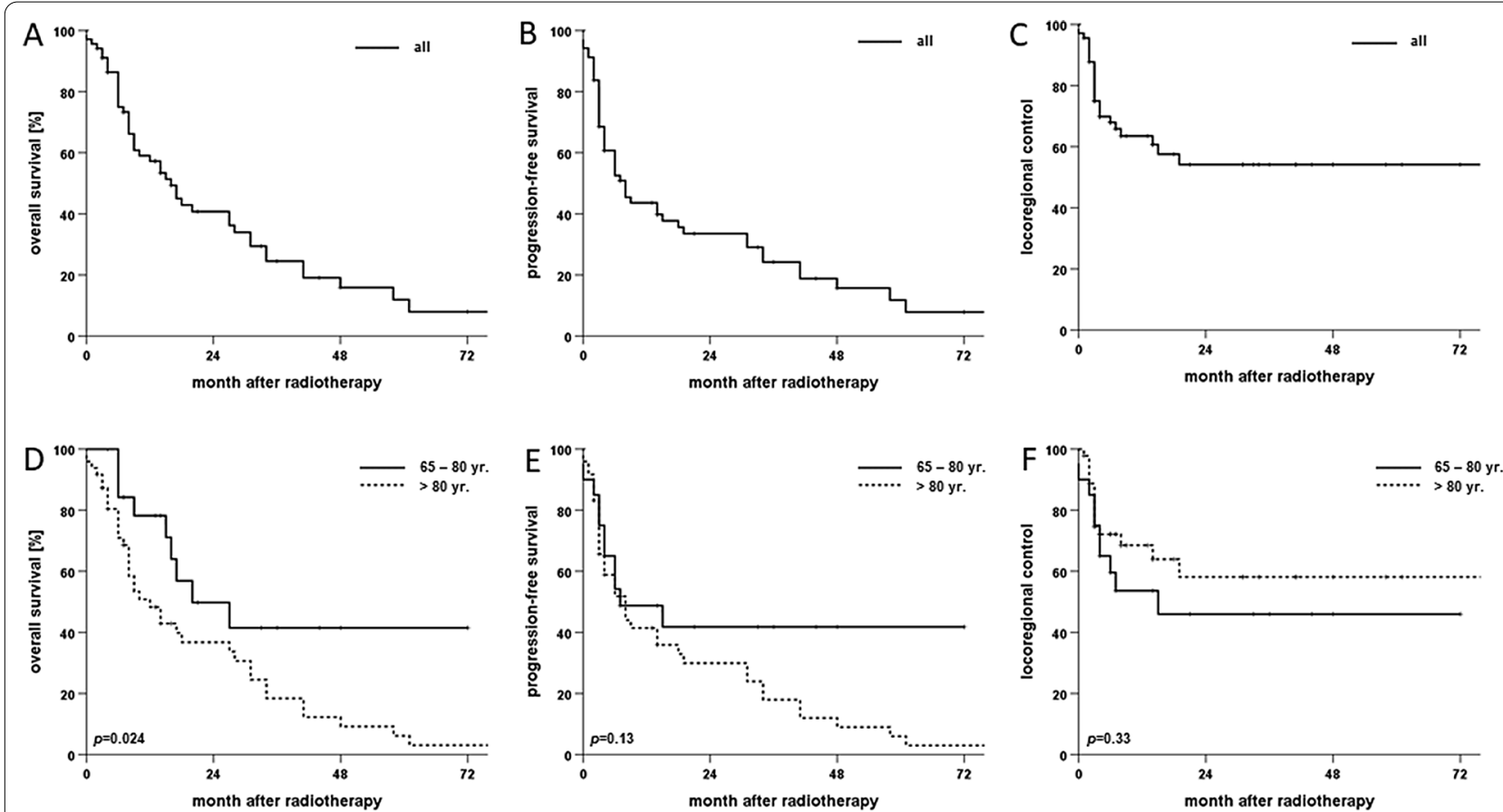

Fig. 2 Kaplan-Meier curves for OS, PFS and LRC of elderly cSCC patients (> 65 years) following radiotherapy $(n=69)$ for the complete cohort $(\mathbf{A}-\mathbf{C})$ and in dependence of age separated at 80 years (D-F), respectively. $P$-values of log-rank tests are shown

lymph node involvement remained the only statistically significant prognostic parameter influencing OS (HR 3.73, CI 1.54-9.03, $p<0.01$ ) (Table 4). In a Cox regression model for the LRC, nodal involvement was the only significant factor worsening LRC (HR 3.72, CI 1.12-12.3, $p=0.03)$. Patients treated for recurrent disease showed a trend to worse LRC (HR 1.71, CI 0.99-2.96, $p=0.06$ ) (Table 4).

\section{Toxicity}

Treatment-related toxicity was moderate in our cohort of elderly cSCC patients undergoing radiotherapy. Only 3 patients (4.3\%) reported any higher-grade acute toxicity (CTCAE grade 3 ) (Tables 5,6$) .81 .2 \%$ of patients $(n=56)$ suffered from at least one mild or moderate (CTCAE grade $1-2)$ adverse event, mostly dermatitis (80\%), dysgeusia (17\%) and xerostomia (17\%). No acute grade 4 or 5 toxicities were observed.

Similarly, the prevalence of chronic toxicities was very low in our patient cohort. Only 22 patients (35\%) experienced at least one mild chronic toxicity (CTCAE grade 1). Reported chronic toxicities were skin-related in 12 patients (19\%), xerostomia in 9 patients $(15 \%)$ and hearing impairments in 4 patients (6\%). Importantly, no grade 2 to 5 chronic toxicities were observed.

\section{Discussion}

In this study, we demonstrated comparably acceptable LRC rates for definitive radiotherapy and adjuvant radiotherapy after incomplete resection. Previous retrospective analyses reported higher LRC rates after definitive photon radiotherapy ranging at almost $90 \%[12,21,22]$. Only the cohort of Cognetta et al. comprised a comparable patient age with a mean of 79 years. In contrast to our study, almost all patients in the studies of Grossi et al. as well as Cognetta et al. exhibited T1 tumors without nodal metastases or high-risk features, as a possible explanation for the favorable outcome. For this low-risk tumors orthovoltage techniques has been used by Grossi and Cognetta. This technique is not available at our department, and could have been used only for a small number of our patients, given the high prevalence of high-risk features. In addition, patients in these reported cohorts were referred to primary radiotherapy, whereas in our cohort, only those patients who were not eligible for surgery were enrolled to receive primary radiotherapy. It has to be noted that the evaluation of local failure is complicated by the commonly displayed field cancerization of heavily sun-damaged areas, where newly occurring tumors in close proximity can hardly be differentiated between recurrence or de-novo cancers [23]. 

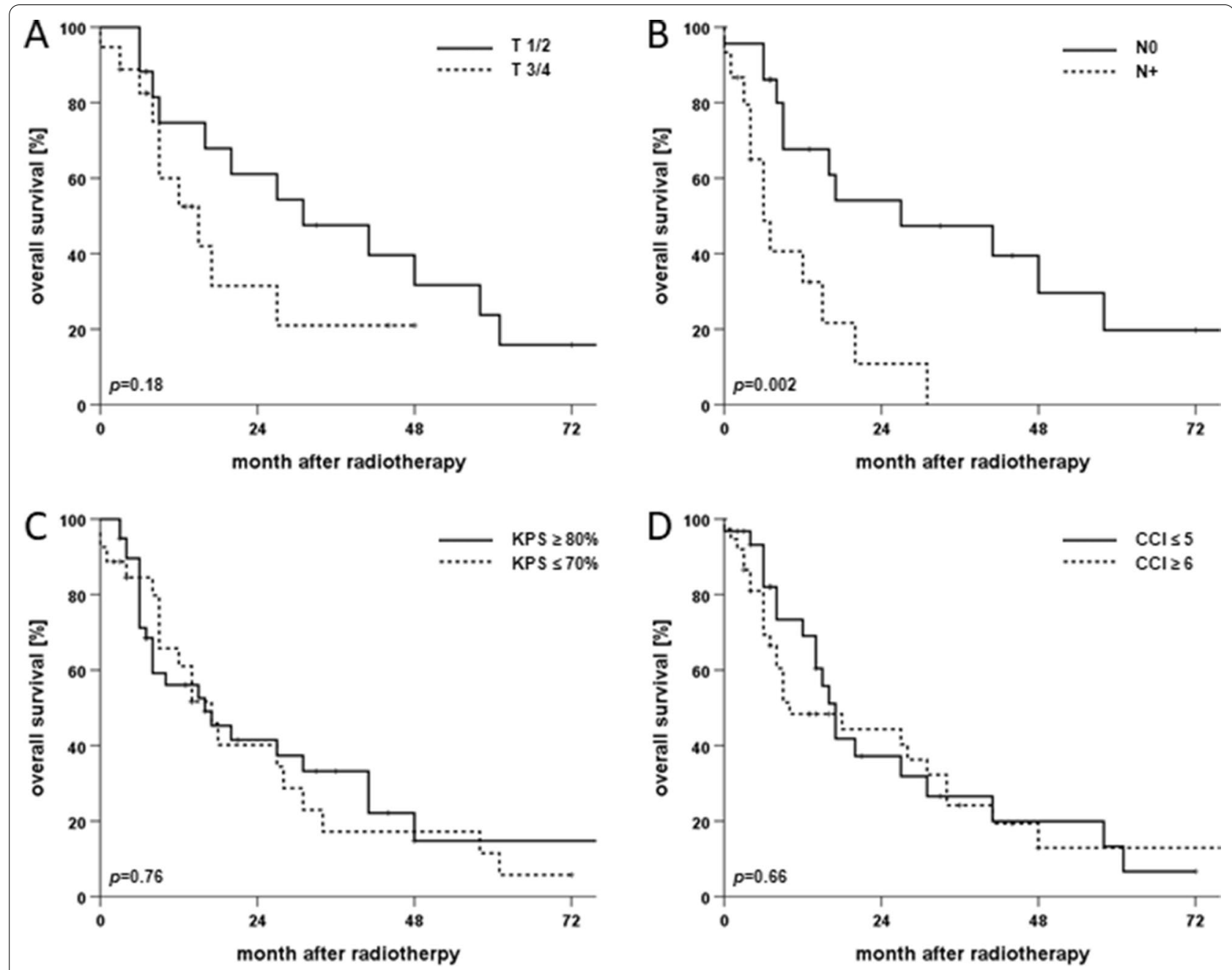

Fig. 3 Kaplan-Meier curves for OS of elderly cSCC patients (> 65 years) after radiotherapy in dependence of T stage (A), N Stage (B), KPS (C) and modified $C C I(D), n=35,37,66$ and 69 , respectively. $P$-values of log-rank tests are displayed

Importantly, we did not detect differences in survival or LRC between patients treated with primary compared to adjuvant radiotherapy. Given the higher prevalence of high-risk features in the adjuvant treatment group (Table 3), this highlights the role of adjuvant treatment even for elderly patients with high-risk features such as positive resection margins, lymph node involvement or recurrent disease. A retrospective study in patients with regional metastatic cSCC from Palme et al. found significantly worse survival after primary radiotherapy compared to multimodal treatment; however, the analysis did not show any data on LRC or detailed patient characteristics [24].
Concerning adjuvant treatment, Sun et al. reported similar LRC to our dataset after surgical resection followed by radiotherapy with around 35\% locoregional failures in a cohort with a median age of 71 years [25]. A further publication by Harris et al. showed an improvement of LRC and OS by adjuvant radiotherapy compared to surgery alone in patients at high risk of tumor recurrence. The reported 2-year OS of around 70\% is notably higher than our results [18]. However, it should be noted that the elderly patient cohort included in their analysis was on average ten years younger, strongly supporting our finding of patient age being the predominant survival factor. 


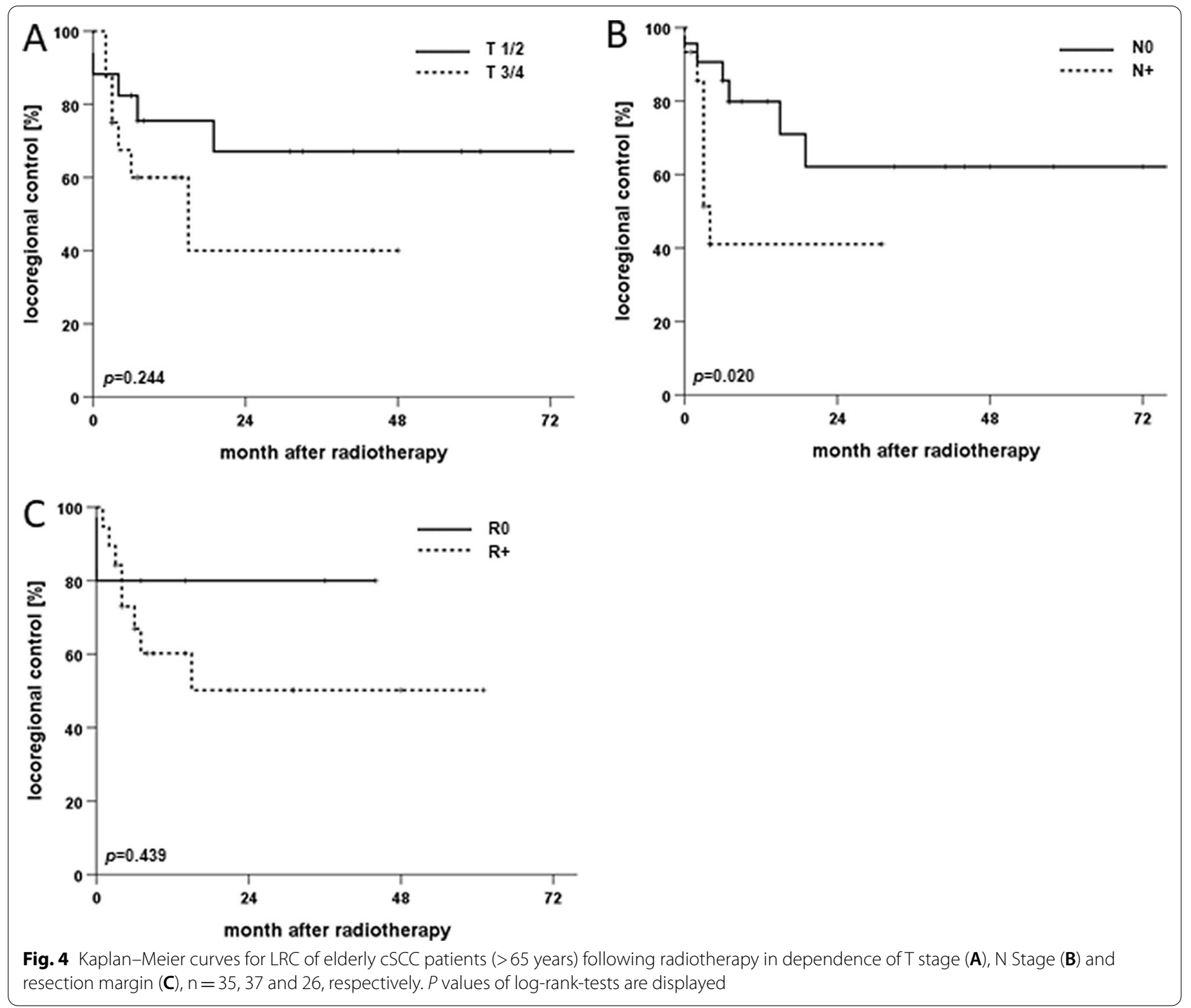

Lymph node involvement revealed itself as the strongest prognostic factor in our cohort. A significant reduction of LRC translated into a significantly reduced OS in our multivariate Cox regression model. Comparable results regarding lymph node involvement have been described by other datasets $[17,26]$. Even after primary surgery followed by adjuvant radiotherapy, the results remain unsatisfactory for patients with nodal metastases, and further systemic treatment may be considered for those patients. PD-1 inhibitors like pembrolizumab and cemiplimab have shown efficacy, but response rates of $50 \%$ or less require further patient stratification [27-29]. In addition, a case series has been suggesting radiotherapy with concomitant pembrolizumab as an alternative for inoperable cSCC [30].

Regarding the importance of LRC for the prognosis of elderly cSCC patients, escalation of radiation treatment doses may provide additional benefits to improve tumor control rates. The current guideline of the ASTRO suggests a variety of conventional and hypofractionated treatment schedules with EQD2 values of up to $77.8 \mathrm{~Gy}$ [20]. Median radiation doses in our cohort in the curative setting were slightly lower than that. Considering the overall low toxicity rates observed in our vulnerable patient cohort, dose escalation may also be a feasible approach even for elderly cSCC patients. Due to the high 
Table 3 Comparison of clinical parameters for radiotherapy subgroups, primary vs. adjuvant and photon vs. electron radiotherapy in elderly cSCC patients

\begin{tabular}{|c|c|c|c|c|}
\hline & \multicolumn{2}{|c|}{ Primary RT $(n=33)$} & \multicolumn{2}{|c|}{ Adjuvant RT $(n=22)$} \\
\hline & $\%$ & $\mathrm{n}$ & $\%$ & $\mathbf{n}$ \\
\hline Mean age & 84.7 & $(66-99)$ & 80.3 & $(70-90)$ \\
\hline $\mathrm{N}+$ & 12.1 & 4 & 22.7 & 5 \\
\hline $\mathrm{R}+$ & - & - & 63.6 & 14 \\
\hline Initial diagnosis & 48.5 & 16 & 31.8 & 7 \\
\hline Local recurrence & 42.4 & 14 & 45.5 & 10 \\
\hline \multirow[t]{3}{*}{ Locoregional recurrence } & 9.1 & 3 & 22.7 & 5 \\
\hline & \multicolumn{2}{|c|}{ Photon RT $(n=46)$} & \multicolumn{2}{|c|}{ Electron RT ( $n=19)$} \\
\hline & $\%$ & $\mathrm{n}$ & $\%$ & $\mathrm{n}$ \\
\hline Mean age & 83.1 & $(66-99)$ & 84.3 & $(70-99)$ \\
\hline $\mathrm{N}+$ & 28.3 & 13 & 5.3 & 1 \\
\hline$R+$ & 32.6 & 15 & 15.8 & 3 \\
\hline Initial diagnosis & 26.1 & 12 & 68.4 & 13 \\
\hline Local recurrence & 50.0 & 23 & 26.3 & 5 \\
\hline Locoregional recurrence & 23.9 & 11 & 5.3 & 1 \\
\hline
\end{tabular}

Table 4 Univariate and multivariate analysis of clinical and pathological parameters regarding OS in elderly cSCC patients receiving radiotherapy

\begin{tabular}{|c|c|c|c|}
\hline Univariate & HR for OS & $\mathrm{Cl} 95 \%$ & $p$-value \\
\hline Age $>80$ years & 2.22 & $1.07-4.60$ & 0.032 \\
\hline $\mathrm{N}+$ & 3.68 & $1.52-8.95$ & 0.004 \\
\hline $\mathrm{R}+$ & 5.38 & $0.70-41.08$ & 0.105 \\
\hline $\mathrm{CCl} \geq 6$ & 1.14 & $0.63-2.05$ & 0.670 \\
\hline$K P S \leq 70 \%$ & 1.10 & $0.60-2.00$ & 0.763 \\
\hline Multivariate & HR for OS & $\mathrm{Cl} 95 \%$ & $p$-value \\
\hline Age $>80$ years & 1.90 & $0.78-4.65$ & 0.159 \\
\hline $\mathrm{N}+$ & 3.73 & $1.54-9.03$ & 0.004 \\
\hline Univariate & HR for LRC & $\mathrm{Cl} 95 \%$ & $p$-value \\
\hline Age $>80$ years & 0.68 & $0.30-1.52$ & 0.351 \\
\hline Tstage & 1.63 & $0.90-2.96$ & 0.108 \\
\hline $\mathrm{R}+$ & 2.22 & $0.28-17.8$ & 0.454 \\
\hline $\mathrm{N}+$ & 3.72 & $1.12-12.3$ & 0.031 \\
\hline total dose (EQD2) & 0.99 & $0.96-1.02$ & 0.496 \\
\hline recurrence vs. initial diagnosis & 1.71 & $0.99-2.96$ & 0.058 \\
\hline$K P S \leq 70 \%$ & 0.90 & $0.39-2.06$ & 0.803 \\
\hline
\end{tabular}

rate of patients with nodal involvement and patients with high risk for nodal spread and consecutive elective nodal irradiation, the majority of our patients has been treated with normofractionated schemes to avoid excessive toxicity. On the other hand, hypofractionated schemes, as mentioned in the ASTRO guideline, could improve 
Table 5 Toxicity results after radiotherapy of elderly patients with CSCC according to the Common Terminology Criteria for Adverse Events (CTCAE) v5.0

\begin{tabular}{lrr}
\hline & $\mathbf{n}$ & $\%$ \\
\hline Acute toxicity $(\mathbf{n}=\mathbf{6 9})$ & 10 & \\
CTCAE 0 & 56 & 14.5 \\
CTCAE 1-2 & 3 & 81.2 \\
CTCAE 3 & 0 & 4.3 \\
CTCAE $\geq$ 4 & & 0.0 \\
Chronic toxicity $(\mathbf{n}=\mathbf{6 2})$ & 47 & \\
CTCAE 0 & 22 & 75.8 \\
CTCAE 1 & 0 & 35.5 \\
CTCAE 2-5 & & 0.0 \\
\hline
\end{tabular}

treatment adherence through reduced overall treatment time especially in the elderly and should be applied whenever safely feasible.

Besides lymph node involvement, age was the second strong prognostic parameter for OS in our analysis. Given the very advanced age of our patient cohort, OS values are likely due to the non-cancer mortality of our elderly patients [30, 31]. Similarly, Harris et al. reported reduced OS and a trend towards reduced LRC in patients older than 70 years treated with adjuvant radiotherapy for cSCC, thus supporting our data [18]. Carter et al. also reported a similar risk of local recurrence, metastasis and disease-specific death between younger and elderly patients, but a significantly higher risk for death of any cause for the elderly population [14].

However, it is generally accepted that the chronological age is commonly of less importance than the biological age, and therefore, other indicators are incorporated into the treatment outcome models as surrogates for biological age such as patient performance or comorbidity burden [32-34]. Unexpectedly, neither performance status nor the burden of comorbidities had a significant influence on OS in our cohort. Although KPS has shown its prognostic value in many cancer entities [35-38], the influence of the performance status on the oncological outcomes in CSCC has not been reported in other patient datasets [12, 15, 17, 25]. Our patient cohort exhibited a relatively good performance status and an overall low burden of comorbidities, especially considering the very advanced age. This may be due to the lack of critical risk factors for cSCCs that also cause significant comorbidities as described for head-and-neck squamous cell carcinomas, where smoking and cumulative alcohol intake play major roles $[38,39]$. KPS and CCI are composite parameters of multiple functional and anamnestic dimensions and therefore constitute feasible surrogate parameters for physical resources and resilience. Cancer therapies like chemotherapy and extensive surgery often severely stress these resources. Radiotherapy for cSCC in our elderly cohort was generally well tolerated with few toxicities. This may be a possible explanation for the lack of influence of KPS and CCI on patient survival.

While our analysis provides insight into the population of elderly cSCC patients treated with radiotherapy and their oncologic outcome, it has certain limitations. The retrospective character may impair access to information about treatment-related toxicity data or comorbidities. Additionally, data on perineural involvement, a reported prognostic factor for decreased survival and LRC, was only available for a small number of patients in our cohort $[13,14]$.

In summary, our analysis of radiotherapy for CSCC of the head-and-neck region in elderly patients indicates acceptable LRC but low OS in this adversely selected cohort. The strongest prognostic factor in the multivariate analysis for OS was lymph node involvement, emphasizing careful pretherapeutic staging. Considering the local disease burden of untreated cSCCs especially for elderly patients, primary radiotherapy constitutes a feasible treatment option even for patients with very advanced age not eligible for surgery. Further prospective studies are needed to corroborate our findings. 
Table 6 Toxicity results consisting various radiotherapy-related adverse reactions according to the Common Terminology Criteria for Adverse Events (CTCAE) v5.0

\begin{tabular}{|c|c|c|c|c|c|c|}
\hline CTCAE & 0 & 1 & 2 & 3 & 4 & 5 \\
\hline \multicolumn{7}{|l|}{ Acute $(n=69)$} \\
\hline Skin toxicity & 13 & 45 & 10 & 1 & 0 & 0 \\
\hline Dysphagia & 62 & 6 & 0 & 1 & 0 & 0 \\
\hline Weight loss & 63 & 4 & 1 & 0 & - & - \\
\hline Nausea & 67 & 2 & 0 & 0 & - & - \\
\hline Mucositis & 53 & 5 & 10 & 1 & 0 & 0 \\
\hline Xerostomia & 57 & 9 & 3 & 0 & - & - \\
\hline Hoarseness & 69 & 0 & 0 & 0 & - & - \\
\hline Dyspnea & 69 & 0 & 0 & 0 & 0 & 0 \\
\hline Dysgeusia & 57 & 11 & 1 & - & - & - \\
\hline Pain & 59 & 5 & 5 & 0 & - & - \\
\hline Cytopenia & 68 & 1 & 0 & 0 & 0 & 0 \\
\hline Otitis & 68 & 0 & 1 & 0 & 0 & 0 \\
\hline Conjunctivitis & 63 & 4 & 2 & 0 & 0 & 0 \\
\hline Infection & 66 & 1 & 2 & 0 & 0 & 0 \\
\hline Hearing loss & 63 & 5 & 1 & 0 & 0 & 0 \\
\hline Hyposmia & 69 & 0 & 0 & 0 & 0 & 0 \\
\hline Neuropathy & 68 & 1 & 0 & 0 & 0 & 0 \\
\hline Alopecia & 67 & 2 & 0 & 0 & 0 & 0 \\
\hline Lymphedema & 67 & 2 & 0 & 0 & 0 & 0 \\
\hline Epiphora & 67 & 2 & 0 & 0 & 0 & 0 \\
\hline Vertigo & 68 & 1 & 0 & 0 & 0 & 0 \\
\hline \multicolumn{7}{|l|}{ Chronic $(n=62)$} \\
\hline Skin toxicity & 55 & 7 & 0 & 0 & 0 & 0 \\
\hline Dysphagia & 61 & 1 & 0 & 0 & 0 & 0 \\
\hline Weight loss & 62 & 0 & 0 & 0 & - & - \\
\hline Nausea & 62 & 0 & 0 & 0 & - & - \\
\hline Mucositis & 62 & 0 & 0 & 0 & 0 & 0 \\
\hline Xerostomia & 53 & 9 & 0 & 0 & - & - \\
\hline Hoarseness & 62 & 0 & 0 & 0 & - & - \\
\hline Dyspnea & 62 & 0 & 0 & 0 & 0 & 0 \\
\hline Dysgeusia & 59 & 3 & 0 & - & - & - \\
\hline Pain & 60 & 2 & 0 & 0 & - & - \\
\hline Cytopenia & 62 & 0 & 0 & 0 & 0 & 0 \\
\hline Renal insufficiency & 62 & 0 & 0 & 0 & 0 & 0 \\
\hline Jaw and dental injuries & 61 & 1 & 0 & 0 & 0 & 0 \\
\hline Neuropathy & 62 & 0 & 0 & 0 & 0 & 0 \\
\hline Hyposmia & 62 & 0 & 0 & 0 & 0 & 0 \\
\hline Alopecia & 62 & 0 & 0 & 0 & 0 & 0 \\
\hline Hearing loss & 58 & 4 & 0 & 0 & 0 & 0 \\
\hline Hyperpigmentation & 57 & 5 & 0 & 0 & 0 & 0 \\
\hline Xerophthalmia & 61 & 1 & 0 & 0 & 0 & 0 \\
\hline Tinnitus & 60 & 2 & 0 & 0 & 0 & 0 \\
\hline Decreased vision & 61 & 1 & 0 & 0 & 0 & 0 \\
\hline Lymphedema & 61 & 1 & 0 & 0 & 0 & 0 \\
\hline Epiphora & 61 & 1 & 0 & 0 & 0 & 0 \\
\hline
\end{tabular}



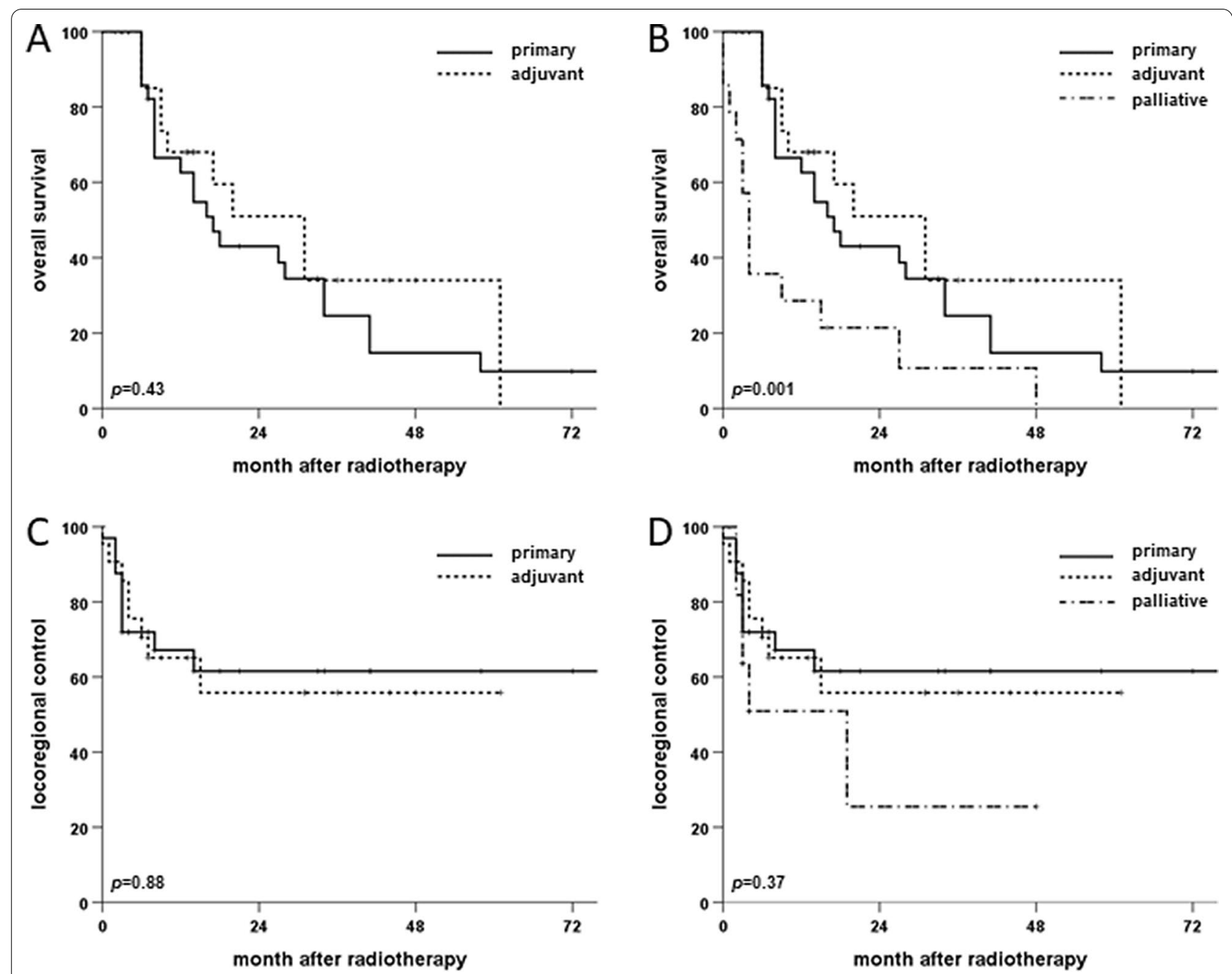

Fig. 5 Kaplan-Meier curves showing OS (A and B) and LRC (C and D) of elderly CSCC patients ( $>65$ years) following primary, adjuvant or palliative radiotherapy $(n=69)$. P-values of log-rank tests are displayed

\section{Supplementary Information}

The online version contains supplementary material available at https://doi. org/10.1186/s13014-021-01832-3.

Additional file 1. Additional information regarding CSCC localizations, reasons for palliative treatment, fractionation regimens and reasons for toxicity-related discontinuation of radiotherapy.

Additional file 2: Figure S1. Kaplan-Meier curves for LRC of elderly CSCC patients (> 65 years) following radiotherapy sorted by tumor localization.

\section{Acknowledgements}

Not applicable.

\section{Authors' contributions}

EH and NHN: Study concept and study design. EH, RK, NHN: Data acquisition, data analysis and data interpretation. EH, AR, NHN: Statistical analysis, manuscript preparation, manuscript editing. EH, AR, RB, TK, TS, EG, CZ, FM, ALG, NHN: Manuscript reviewed. All authors read and approved the final manuscript.

\section{Funding}

Open Access funding enabled and organized by Projekt DEAL. This research did not receive any specific grant from funding agencies in the public, commercial, or not-for-profit sectors.

\section{Availability of data and materials}

The datasets generated and/or analysed during the current study are not publicly available due to patients information privacy but are available from the corresponding author on reasonable request.

\section{Declarations}

Ethics approval and consent to participate

Ethics approval of the University of Freiburg ethics committee reference number EKVotum 551/18. Consent to participate not applicable.

\section{Consent for publication}

Not applicable.

\section{Competing interests}

The authors declare that they have no competing interests. 


\begin{abstract}
Author details
'Department of Radiation Oncology, University of Freiburg - Medical Center, Robert-Koch-Str. 3, 79106 Freiburg, Germany. ${ }^{2}$ German Cancer Research Center (Dkfz), German Cancer Consortium (DKTK) Partner Site Freiburg, Neuenheimer Feld 280, 69120 Heidelberg, Germany. ${ }^{3}$ Department of Dermatology and Venereology, University of Freiburg - Medical Center, Hauptstr. 7, 79104 Freiburg, Germany.
\end{abstract}

Received: 27 January 2021 Accepted: 1 June 2021

Published online: 12 June 2021

\section{References}

1. Bray F, et al. Global cancer statistics 2018: GLOBOCAN estimates of incidence and mortality worldwide for 36 cancers in 185 countries. CA Cancer J Clin. 2018;68(6):394-424.

2. World Health Organisation. Ultraviolet (UV) radiation and skin cancer. Geneva:WHO; 2017.https://www.who.int/news-room/q-a-detail/radia tion-ultraviolet-(uv)-radiation-and-skin-cancer. Accessed 2 Jan 2021.

3. Stern RS. Prevalence of a history of skin cancer in 2007: results of an incidence-based model. Arch Dermatol. 2010;146(3):279-82.

4. Muzic JG, et al. Incidence and trends of basal cell carcinoma and cutaneous squamous cell carcinoma: a population-based study in Olmsted County, Minnesota, 2000 to 2010. Mayo Clin Proc. 2017;92(6):890-8.

5. Waldman A, Schmults C. Cutaneous squamous cell carcinoma. Hematol Oncol Clin N Am. 2019;33(1):1-12

6. Maubec E. Update of the management of cutaneous squamous-cell carcinoma. Acta Derm Venereol. 2020;100(11):adv0143.

7. Leibovitch I, et al. Cutaneous squamous cell carcinoma treated with Mohs micrographic surgery in Australia I. Experience over 10 years. J Am Acad Dermatol. 2005;53(2):253-60

8. Chren MM, et al. Tumor recurrence 5 years after treatment of cutaneous basal cell carcinoma and squamous cell carcinoma. J Invest Dermatol. 2013;133(5):1188-96.

9. Karia PS, Han J, Schmults CD. Cutaneous squamous cell carcinoma: estimated incidence of disease, nodal metastasis, and deaths from disease in the United States, 2012. J Am Acad Dermatol. 2013;68(6):957-66.

10. Schmults $C D$, et al. Factors predictive of recurrence and death from cutaneous squamous cell carcinoma: a 10-year, single-institution cohort study. JAMA Dermatol. 2013;149(5):541-7.

11 Clark RR, Soutar DS. Lymph node metastases from auricular squamous cell carcinoma. A systematic review and meta-analysis. J Plast Reconstr Aesthet Surg. 2008:61(10):1140-7.

12 Rowe DE, Carroll RJ, Day CL Jr. Prognostic factors for local recurrence, metastasis, and survival rates in squamous cell carcinoma of the skin, ear, and lip. Implications for treatment modality selection. J Am Acad Dermatol. 1992;26(6):976-90.

13. Ross AS, et al. Diameter of involved nerves predicts outcomes in cutaneous squamous cell carcinoma with perineural invasion: an investigatorblinded retrospective cohort study. Dermatol Surg. 2009:35(12):1859-66.

14. Carter JB, et al. Outcomes of primary cutaneous squamous cell carcinoma with perineural invasion: an 11-year cohort study. JAMA Dermatol. 2013;149(1):35-41.

15. Thompson AK, et al. Risk factors for cutaneous squamous cell carcinoma recurrence, metastasis, and disease-specific death: a systematic review and meta-analysis. JAMA Dermatol. 2016;152(4):419-28.

16. Wang DM, et al. Association of nodal metastasis and mortality with vermilion vs cutaneous lip location in cutaneous squamous cell carcinoma of the lip. JAMA Dermatol. 2018;154(6):701-7.

17. Veness MJ, et al. Surgery and adjuvant radiotherapy in patients with cutaneous head and neck squamous cell carcinoma metastatic to lymph nodes: combined treatment should be considered best practice. Laryngoscope. 2005:115(5):870-5.

18. Harris $\mathrm{BN}$, et al. Association of adjuvant radiation therapy with survival in patients with advanced cutaneous squamous cell carcinoma of the head and neck. JAMA Otolaryngol Head Neck Surg. 2019;145(2):153-8.
19. Jambusaria-Pahlajani A, et al. Surgical monotherapy versus surgery plus adjuvant radiotherapy in high-risk cutaneous squamous cell carcinoma: a systematic review of outcomes. Dermatol Surg. 2009;35(4):574-85.

20. Likhacheva A, et al. Definitive and postoperative radiation therapy for basal and squamous cell cancers of the skin: executive summary of an american society for radiation oncology clinical practice guideline. Pract Radiat Oncol. 2020;10(1):8-20.

21. Grossi Marconi D, et al. Head and neck non-melanoma skin cancer treated by superficial X-ray therapy: an analysis of 1021 cases. PLoS ONE. 2016;11(7):e0156544.

22. Cognetta $A B$, et al. Superficial $x$-ray in the treatment of basal and squamous cell carcinomas: a viable option in select patients. J Am Acad Dermatol. 2012;67(6):1235-41.

23. Slaughter DP, Southwick HW, Smejkal W. Field cancerization in oral stratified squamous epithelium; clinical implications of multicentric origin. Cancer. 1953;6(5):963-8.

24. Palme CE, et al. Extent of parotid disease influences outcome in patients with metastatic cutaneous squamous cell carcinoma. Arch Otolaryngol Head Neck Surg. 2003;129(7):750-3.

25. Sun $\mathrm{L}$, et al. Association of disease recurrence with survival outcomes in patients with cutaneous squamous cell carcinoma of the head and neck treated with multimodality therapy. JAMA Dermatol. 2019;155(4):442-7.

26. Ch'ng S, et al. Parotid and cervical nodal status predict prognosis for patients with head and neck metastatic cutaneous squamous cell carcinoma. J Surg Oncol. 2008;98(2):101-5.

27. Grob JJ, et al. Pembrolizumab monotherapy for recurrent or metastatic cutaneous squamous cell carcinoma: a single-arm phase II trial (KEYNOTE-629). J Clin Oncol. 2020;38(25):2916-25.

28. Migden MR, et al. PD-1 blockade with cemiplimab in advanced cutaneous squamous-cell carcinoma. N Engl J Med. 2018;379(4):341-51.

29. Vaidya $P$, et al. Concurrent radiation therapy with programmed cell death protein 1 inhibition leads to a complete response in advanced cutaneous squamous cell carcinoma. JAAD Case Rep. 2019;5(9):763-6.

30. DeSantis CE, et al. Cancer statistics for adults aged 85 years and older, 2019. CA Cancer J Clin. 2019:69(6):452-67.

31. Sprave T, et al. Radiotherapy for nonagenarians: the value of biological versus chronological age. Radiat Oncol. 2020;15(1):113.

32. Grenman R, et al. Treatment of head and neck cancer in the elderly: European Consensus (panel 6) at the EUFOS Congress in Vienna 2007. Eur Arch Otorhinolaryngol. 2010;267(10):1619-21.

33. Rühle $A$, et al. The value of laboratory parameters for anemia, renal function, systemic inflammation and nutritional status as predictors for outcome in elderly patients with head-and-neck cancers. Cancers. 2020;12(6):1698

34. Ruhle A, et al. Radiation-induced toxicities and outcomes after radiotherapy are independent of patient age in elderly salivary gland cancer patients: results from a matched-pair analysis of a rare disease. Eur Arch Otorhinolaryngol. 2020:278(7):2537-548.

35. Buccheri G, Ferrigno D, Tamburini M. Karnofsky and ECOG performance status scoring in lung cancer: a prospective, longitudinal study of 536 patients from a single institution. Eur J Cancer. 1996;32A(7):1135-41.

36. Carson KA, et al. Prognostic factors for survival in adult patients with recurrent glioma enrolled onto the new approaches to brain tumor therapy CNS consortium phase I and II clinical trials. J Clin Oncol. 2007:25(18):2601-6

37. Sperduto PW, et al. Summary report on the graded prognostic assessment: an accurate and facile diagnosis-specific tool to estimate survival for patients with brain metastases. J Clin Oncol. 2012;30(4):419-25.

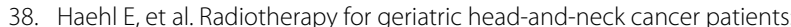
what is the value of standard treatment in the elderly? Radiat Oncol. 2020;15(1):31

39. Paleri $V$, et al. Comorbidity in head and neck cancer: a critical appraisal and recommendations for practice. Oral Oncol. 2010:46(10):712-9.

\section{Publisher's Note}

Springer Nature remains neutral with regard to jurisdictional claims in published maps and institutional affiliations. 